\title{
Vaping during the COVID-19 lockdown period in Belgium
}

\author{
Karolien Adriaens ${ }^{1}$, Dinska Van Gucht ${ }^{2}$, Sven Van Lommel ${ }^{3}$, Frank Baeyens ${ }^{1}$ \\ 1 University of Leuven \\ 2 Thomas More College \\ 3 Universitair Ziekenhuis Leuven
}

Funding: The author(s) received no specific funding for this work.

Potential competing interests: The author(s) declared that no potential competing interests exist.

\section{Abstract}

Background: Due to the Corona Virus Disease 2019 (COVID-19), the Belgian government has set out a range of measures to prevent the spread of the virus. One measure included closing all non-food shops, including vape shops.

Methods: A retrospective online questionnaire was used to investigate the impact of closing the vape shops on the vaping and/or smoking behavior of current vapers. Results: The sample $(n=202)$ reached consisted of $70 \%$ exclusive vapers, $29 \%$ dual users and $1 \%$ no-product users. Over half (55\%) of participants was in need to buy eliquid during the lockdown, with a small majority being able to buy e-liquids - mostly with their usual nicotine concentrations, flavor or brand - , but as much as 39\% of them running out of e-liquid. Those buying e-liquid mainly did so by making purchases via foreign online webshops. A similar pattern was observed with respect to purchasing hardware, with about half (47\%) of participants reporting hardware availability and with a small majority (53\%) reporting hardware unavailability. Of those indicating that hardware was not available, 38\% ran out of a properly functioning e-cigarette. A nontrivial minority was forced to consume e-liquids with another nicotine concentration, flavor or brand than usual. One seventh of exclusive vapers relapsed partly or completely to smoking during the lockdown. The main reasons for changing vaping and/or smoking behavior included the unavailability of e-liquid with nicotine, the unavailability of hardware, and stress/worries about COVID-19.

Conclusion: The majority of vapers succeeded in maintaining their vaping behavior as usual, highly likely due to (illegally) buying consumables online. Nevertheless, for a minority the lockdown period resulted in unintended consequences and these vapers relapsed (completely) to smoking. Even during periods of lockdown, smokers and vapers should be able to purchase low(er)-risk alternatives to smoking, for example e- 
cigarettes.

\section{INTRODUCTION}

Early March 2020, the first confirmed infections with the Corona Virus Disease 2019 (COVID-19) in Belgium have been recorded. In order to prevent the spread of the virus, the government has set out a range of measures including closing stores (exceptions: food stores, newsagents and pharmacies) for a period of almost two months (March 18th until May 10th). Consequently, all vape shops were closed and vapers could no longer purchase consumables (e.g., e-liquids, batteries, coils) for their electronic cigarettes (e-cigarettes) in brick-and-mortar vape shops. Some newsagents and some food stores, however, offered a limited product range of e-liquids and e-cigarettes, or temporarily expanded their range (sometimes in cooperation with closed brick-andmortar vape shops). Buying consumables online is complicated in Belgium due to the fact that the online sale of e-cigarettes and e-liquids is prohibited. ${ }^{[1]}$ More specifically this prohibition implies that Belgian vape shop owners cannot sell their consumables online (nor do they do so), that foreign webshops cannot sell to Belgian vapers as this is a criminal offence, and that customs can (and occasionally do) seize consumables ordered abroad. The online purchase of consumables itself is not punishable, however. In contrast, smokers still had the opportunity to unrestrictedly buy their cigarettes in food stores or from newsagents during the lockdown period. The severely restricted availability of low(er)-risk alternatives (i.e. e-cigarettes) for smoking during lockdown, could potentially have negative consequences on one's vaping behavior, including the risk of (re)lapse to smoking. Therefore, the purpose of this study was to investigate to what extent the lockdown has had an impact on vaping and/or smoking behavior of current vapers (including also dual users of e-cigarettes and tobacco cigarettes). In the current study we used a retrospective online questionnaire to investig ate the impact of closed vape shops on changes in vaping and/or smoking behavior in current Belgian vapers.

\section{METHODS}

\section{Participants}

Participants were recruited through social media. The sample reached consisted of current adult (+18 years) Dutch or French speaking Belgian vapers, including exclusive vapers, and dual users of e-cigarettes and tobacco cigarettes. A total of 241 participants started filling out the online questionnaire, with only two (1\%) not giving their consent for participation. The questionnaire was fully completed by 202 participants (85\%).

\section{Procedure}


The current study is in accordance with the General Data Protection Regulation (GDPR) and was approved by the Societal and Social Ethics Committee of the University of Leuven (G-2020-1960).

The link to the online questionnaire, made in Qualtrics, was distributed through social media (T witter, Facebook, "Belgische Damp Bond / Union Belge pour La Vape") from May 25th until June 8th. After clicking on the link, participants firstly received general information about the study and were asked to give their consent to participate. When they did not give their consent, participants were thanked for their interest and the questionnaire was finished. Participants who gave their consent, were provided with the questionnaire, thanked at the end and finally were given the opportunity to enter their email address if they wanted to receive information about the results of the study.

\section{Materials and outcome measures \\ Questionnaire}

The questionnaire started with assessing sociodemographic variables like age, gender, highest obtained degree, occupation, marital status, net income per month and nationality (all predefined categories, except age). Next, participants reported on their smoking and vaping status before the lockdown period (i.e. before March 18th) by indicating how frequently (daily, weekly, monthly, not) they were vaping and/or smoking. Subsequently, participants were asked about their stock of consumables (e-liquids and service/hardware), their need of purchasing consumables, their ability to purchase these consumables, and how and where they had made such purchases. This was followed by questions that focused on the type of e-liquid (nicotine concentration) they were using and their vaping frequency (more, less, same as usual, quit vaping) during the lockdown period. Finally, the questionnaire investigated whether any changes had occurred during the lockdown period regarding the vaping and smoking behavior, separately for exclusive vapers and dual users, and what the reasons were for these changes. ${ }^{[2]}$

\section{Statistical analyses}

We used descriptive analyses, including frequencies and proportions, to describe the obtained results. Additionally, cross-tabulations were used to get a more detailed picture of specific subgroups (e.g., not being able to purchase supplies) of the sample and their changes in vaping and/or smoking behavior. All analyses were conducted using Statistica, version 13.

\section{RESULTS}

Participants were on average 39 years old $(S D=9.89)$ and were mainly middle-class, full- 
time working males (all details are presented in Table 1). Before the lockdown period, only one participant (1\%) was not smoking nor vaping, $70 \%$ of the sample were exclusive vapers ( $n=142)$ and $29 \%$ were dual users $(n=59)$. Among those dual users, the majority $(66 \%, 39 / 59)$ smoked daily and the others smoked weekly $(19 \%, 11 / 59)$ or monthly $(15 \%$, 9/59). Vapers (including dual users) were vaping daily (96\%).

About half $(45 \%, 90 / 202)$ of the sample had sufficient e-liquid in stock during the lockdown period, and around one third $(31 \%, 28 / 90)$ of those reported, however, buying some extra e-liquid. The other two thirds $(69 \%, 62 / 90)$ were not in need of e-liquid nor bought extra e-liquid, but 57\% (35/62) of these participants did indicate that they would not have been able to purchase e-liquid if it were needed. In contrast, 55\% (112/202) of participants did not have enough e-liquid to bridge the vape shop lockdown period. The majority $(57 \%, 64 / 112)$ of them was able to make purchases but $39 \%(44 / 112)$ ran out of e-liquid. The remaining four $(4 \%, 4 / 112)$ participants reported not being able to buy eliquid and that this was not needed. The majority of the participants who did buy e-liquid ( $n=92$ ) were able to purchase e-liquid with their usual nicotine concentration (89\%, $82 / 92)$, their usual flavor $(65 \%, 60 / 92)$, and of their usual brand $(67 \%, 62 / 92)$. All details are presented in Table 2 . Most of them $(52 \%, 48 / 92)$ bought e-liquid via an online webshop (outside Belgium), others in a brick-and-mortar vape shop on demand (24\%, 22/92), at a brick-and-mortar vape shop pickup point (15\%, 14/92), from a newsagent $(14 \%, 13 / 92)$, via friends $(7 \%, 6 / 92)$ or in a gas station $(5 \%, 5 / 92)$, see Figure 1. We also asked participants to indicate their ability to receive service or purchase any hardware (e.g., e-cigarettes, batteries, coils, atomizers, ...) and their need for doing so during the lockdown period (see Table 2). About half of participants (47\%, 95/202) indicated that hardware could be purchased, and nearly half $(40 \%, 38 / 95)$ of these participants did so. Among the other half of participants (53\%, 107/202) who reported that hardware was not available, less than half $(38 \%, 41 / 107)$ ran out of properly functioning hardware and the majority $(62 \%, 66 / 107)$ was not in need for hardware. Among those who were able and did buy some hardware $(n=38)$, the majority (63\%, 24/38) did so via an online webshop (outside Belgium) or in a brick-and-mortar vape shop on demand $(24 \%, 9 / 38)$ or from a newsagent $(18 \%, 7 / 38)$, see Figure 1.

Regarding changes in vaping and/or smoking behavior during the lockdown period, most of the participants $(85 \%, 172 / 202)$ consumed e-liquids with the same nicotine concentration (including nicotine free e-liquids) as usual. A minority was forced to use lower nicotine concentrations (7\%, 15/202), higher nicotine concentrations (5\%, 9/202), or nicotine free e-liquids $(2 \%, 4 / 202)$ compared to their usual nicotine concentrations. Two participants (1\%) were even obliged to consume e-liquid with nicotine whereas they normally vaped nicotine free e-liquids. Similarly, the majority of participants reported 
vaping as much as usual $(60 \%, 121 / 202)$, whereas a fourth vaped more $(25 \%, 51 / 202)$ and a minority less $(8 \%, 17 / 202)$ than usual. Few $(6 \%, 13 / 202)$ had quit vaping during the lockdown period. Interestingly, all participants who indicated to have quit vaping ( $n=13)$, reported they ran out of e-liquid during the lockdown, and most of them $(77 \%, 10 / 13)$ also ran out of a properly functioning e-cigarette. Among participants who (temporarily or permanently) ran out of e-liquid $(n=44)$, one third $(34 \%, 15 / 44)$ vaped as much as usual, 20\% (9/44) vaped more, 16\% (7/44) vaped less, and 30\% (13/44) quit vaping completely.

Additionally, we also asked both exclusive vapers and dual users how their vaping and/or smoking behavior changed during the lockdown, see Table 3. The majority of exclusive vapers $(85 \%, 121 / 142)$ continued with exclusive vaping. A minority combined vaping with smoking tobacco $(7 \%, 10 / 142)$ or quit vaping and started smoking tobacco $(7 \%$, 10/142), and only one participant (1\%) quit vaping without starting to smoke tobacco. Additionally, among those who indicated they became a dual user $(n=10)$, the majority $(60 \%, 6 / 10)$ ran out of e-liquid, but had sufficient hardware in stock $(60 \%, 6 / 10)$. Of those who became exclusive smokers $(n=10)$, all $(100 \%, 10 / 10)$ ran out of e-liquid and almost all $(90 \%, 9 / 10)$ ran out of a properly functioning e-cigarette. Among dual users ( $n=59)$, the majority $(68 \%, 40 / 59)$ continued using both products after the lockdown, one fifth $(25 \%, 15 / 59)$ became an exclusive vaper, three dual users (5\%) quit using both products, and only one participant (2\%) became an exclusive smoker (and thus quit vaping), see Table 3. More specifically, the majority of dual users reported smoking more $(42 \%$, $25 / 59$ ) and vaping as much as usual $(41 \%, 24 / 59)$, see Table 4 for more details. A majority of participants $(61 \%, 124 / 202)$ reported no changes in vaping and/or smoking behavior. Among those that did change their behaviors (39\%, 78/202), the main reasons included: e-liquid with nicotine was not available $(56 \%, 44 / 78)$, hardware was not available (49\%, 38/78), stress and worries about COVID-19 (45\%, 35/78), flavor was not available $(10 \%, 31 / 78)$, the belief that nicotine provides protection against COVID-19 $(14 \%, 11 / 78)$, the belief that the e-cigarette provides protection ag ainst COVID-19 $(10 \%, 8 / 78)$, the belief that smoking tobacco results in a higher risk infection with COVID-19 (8\%, 6/78), and the fact that nicotine free e-liquid was not available $(6 \%, 5 / 78)$, see Figure 2 .

\section{DISCUSSION}

One measure taken by the Belgian government to prevent the spread of COVID-19 was closing all non-food shops, including the vape shops. The current online retrospective questionnaire study shows that, during this lockdown period, most vapers continued vaping and that even one fifth of dual users became exclusive vapers. Thus, although the brick-and-mortar vape shops were closed and there is a ban on the online sale of e- 
cig arettes in Belgium, most vapers have been able to purchase consumables (e-liquid, hardware) as usual and when needed. Nevertheless, 11\%, 35\% and 33\% was forced to buy e-liquids with another nicotine concentration, another flavor, or another brand as usual, respectively. Although we cannot determine if the vapers reached were already buying consumables online before the lockdown period (as this has not been questioned), it is not unlikely that this was the case as we made use of a convenience sample of websavvy vapers. By doing so, it is most likely that we reached vapers who were familiar with making online purchases. As the online sale of consumables in Belgium is prohibited, but vapers were still able to make online purchases, this implies two things: 1) that foreign webshops have sold illeg ally to Belgian vapers while taking the risk to be punished for doing so, and 2) that the ban on the import of consumables purchased online is not enforced. Due to this minimal enforcement of the legislation, vapers were able to obtain e-liquid/hardware during the lockdown period.

It seems that being able to purchase consumables online has, for many vapers, served as a kind of protective factor in remaining smoking abstinent, and thus not relapsing to smoking. On the other hand, if the legislation would have been properly enforced, these vapers would not have been able to obtain consumables that easily and relapse to smoking rates would most probably have been higher. Nevertheless, for those vapers who are not familiar with buying online or do not take such risks of buying consumables online, closing vape shops during a lockdown results in a decrease in the availability of consumables. The limited availability of low(er)-risk alternatives for smoking could consequently result in an increase in smoking behavior.

It should be stressed that for a non-trivial minority of participants the lockdown period indeed has resulted in combining vaping with (more) smoking, or even quitting vaping completely and relapsing to smoking. One seventh of exclusive vapers started smoking next to vaping (with $60 \%$ running out of e-liquid, but having a properly functioning ecigarette) and or switched completely to exclusive cigarette smoking (with all of them running out of e-liquid and $90 \%$ running out of a properly functioning e-cigarette), and the majority of dual users remained using both products while smoking more than usual. Only a minority of dual users switched completely to vaping. Sciensano, ${ }^{[3]}$ a federal scientific institution and federal research centre for public health, animal health and food safety in Belgium, has also found an increase in smoking prevalence (19.8\% in 2018 vs. 21.9\% during lockdown 2020) during the lockdown. Among smokers, around one third also reported an increase in the amount of cigarettes smoked. ${ }^{[3]}$ Although only a minority of the current sample relapsed to smoking, extrapolation to the Belgian population (approximately 9,500,000 citizens aged 15 year and older) ${ }^{[4]}$ and translating this to absolute numbers may be instructive to estimate the number of vapers that were 
potentially neg atively affected by the lockdown measure in Belgium. Therefore, we combined our results with the most recent Belgian vaping prevalence data. ${ }^{[5]}$ According to IPSOS, $10 \%$ (950,000 people) of the population is vaping at least once in a while, with $3 \%$ (285,000 people) vaping daily. Our sample mainly consisted of daily vapers of whom $56 \%$ did not have enough e-liquid during the lockdown and of whom 39\% ran out of eliquid. This would amount, in absolute numbers, to 159,600 and 62,244 Belgian daily vapers, respectively. A total of $6 \%$ quit vaping, which would translate to 17,100 daily vapers. IPSOS ${ }^{[5]}$ further reports that $41 \%$ of all vapers are exclusive vapers $(389,500$ people). In our sample, $7 \%$ of exclusive vapers relapsed completely to smoking and 7\% became a dual user of e-cigarettes and tobacco cigarettes. This would ag ain translate to 27,265 people having completely and the same number having partly relapsed to smoking. Even though our sample may not be representative for the general (vaping) population, this mathematical exercise clearly highlights the importance of considering the unintended consequences of closing vape shops and denying access to low(er)-risk products to smokers, while cigarettes remain easily available during lockdown periods. In contrast to the relapse rates that we observed, Caponnetto and colleagues ${ }^{[6]}$ have found that Italian exclusive smokers decreased their cigarette consumption and exclusive vapers showed a similar vaping behavior as before the lockdown. It is not clear what factors could potentially explain these differences. Nevertheless, a likely explanatory candidate is that Italy is one of the countries that, despite original plans to do so, in the end did not close the vape shops, such that vapers (more) easily could access their consumables during the lockdown period compared to Belgian vapers. There are differences between EU-countries with regard the opening or closing of vape shops during the lockdown periods, for example, vape shops were open in Finland, France, Switzerland, Denmark, Italy, Romania, Norway, Netherlands, Cyprus, and Sweden. In the countries were brick-and-mortar vape shops were closed (UK, Ireland, Spain, and Germany), the online sale of consumables was allowed, in contrast to Belgium. In the longer term, research is needed to investig ate the effects of these different regulatory decisions on smoking and/or vaping behavior. It is without any doubt that the current results should be seen in light of the following limitations. Firstly, we used a convenience sample of current vapers, which may not be representative for the general population of vapers. Secondly, although we distributed the online questionnaire on social media, the response rate was relatively low resulting in a small, mainly male sample. Thirdly, as is true for most questionnaire-based studies, there was no biological verification of self-reported smoking and/or vaping status.

\section{CONCLUSION}


Carrying out lockdown measures to curb a pandemic can (negatively) impact specific behaviors of specific groups of people. Related to vaping, it is critical to rethink the potential unintended consequences of closing vape shops while there is also a ban on the online sale of e-cigarettes. Smokers and vapers should be provided with the possibility to purchase low(er)-risk alternatives for smoking, even during a lockdown. Denying this opportunity can result in an increase in smoking prevalence, especially when tobacco cigarettes are easily available and the lack of available e-liquids/hardware, and exposure to toxic substances due to the lack of maintenance of e-cigarettes.

\section{References}

1. ^ Federal Public Services, health, food chain safety and environment. (2016).

$$
\text { Notification of e-cigarettes.. }
$$

2. ^ Konstantinos Farsalinos, Anastasia Barbouni, Konstantinos Poulas, Riccardo Polosa, Pasquale Caponnetto, Raymond Niaura. (2020). Current smoking, former smoking, and adverse outcome among hospitalized COVID-19 patients: a systematic review and meta-analysis. Therapeutic Advances in Chronic Disease, vol. 11, 204062232093576. doi:10.1177/2040622320935765.

3. a, b Sciensano. (2020). Tweede COVID-19 gezondheidsenquête: Eerste resultaten..

4. ^ STATBEL. (2017). Structuur van de bevolking..

5. a, b IPSOS. (2019). Rookenquête 2019: Een rapport voor Stichting tegen Kanker, uitgevoerd door IPSOS Belgium..

6. ^ Pasquale Caponnetto, Lucio Inguscio, Claudio Saitta, Marilena Maglia, Francesca Benfatto, Riccardo Polosa. (2020). Smoking behavior and psychological dynamics during COVID-19 social distancing and stay-at-home policies: A survey. Health Psych Res, vol. 8 (1). doi:10.4081/hpr.2020.9124. 